\title{
Algorithm of Classroom Teaching Quality Evaluation Based on Markov Chain
}

\author{
Tongqing Yuan \\ School of Educational Science, Anhui Normal University, Wuhu 241000, Anhui, China \\ Correspondence should be addressed to Tongqing Yuan; asdytq@mail.ahnu.edu.cn
}

Received 24 March 2021; Accepted 11 June 2021; Published 22 June 2021

Academic Editor: Zhihan Lv

Copyright ( $\odot 2021$ Tongqing Yuan. This is an open access article distributed under the Creative Commons Attribution License, which permits unrestricted use, distribution, and reproduction in any medium, provided the original work is properly cited.

\begin{abstract}
The Markov chain model teaching evaluation method is a quantitative analysis method based on probability theory and stochastic process theory, which establishes a stochastic mathematical model to analyse the quantitative relationship in the change and development process of real activities. Applying it to achieve a more comprehensive, reasonable, and effective evaluation of the classroom teaching quality of college teachers is of positive significance for promoting the continuous improvement of the teaching level of teachers and the teaching quality of schools. Therefore, after an in-depth study of Markov chain algorithm theory, this research proposes an improved Markov chain hybrid teaching quality evaluation model and designs comparative experiments and applies it to the hybrid teaching quality evaluation system of universities, designs a corresponding hybrid teaching quality evaluation model, and finally verifies its effectiveness through experiments. The mathematical model of mixed classroom teaching quality evaluation given in this research focuses on the development and change of the teaching process. For the teaching process that is closely related to the causality of teaching quality, the model established in this paper is more objective and reasonable for evaluating the quality of teaching.
\end{abstract}

\section{Introduction}

Classroom teaching quality evaluation is an important link in the process of teaching management. Continuously improving the objectivity and reliability of classroom teaching quality evaluation data is an important way and means to improve the quality of teaching evaluation. The establishment of teaching quality evaluation is to construct the functional relationship between the teaching quality evaluation index and the teaching effect. Teaching quality evaluation is based on the requirements of teaching goals and teaching principles, formulating scientific evaluation standards, systematically collecting information, measuring the process and results of teaching and learning activities, and giving value judgments [1]. Classroom teaching is the basic form and core link of teaching work, and it plays a decisive role in improving the quality of teaching.

There are many methods for evaluating the quality of classroom teaching. Since the content involved in teaching quality evaluation is more qualitative and less quantitative, only qualitative standards can be given, and the standards are more flexible. In addition, the evaluators have deviations in their grasp of the standards and subjective reasons, which brings a certain degree of difficulty to the evaluation of teaching quality. At present, colleges usually adopt methods such as absolute evaluation method, rating method, relative evaluation method, comment method, and comprehensive scoring method. These algorithms are more scientific and persuasive than qualitative methods, but there are still strong professional problems, and it is difficult for many nonprofessionals to understand and use them objectively and reasonably. At the same time, the stability of these algorithms needs to be improved, and the reliability of the results cannot be analysed $[2,3]$. Therefore, how to construct a new teaching quality evaluation model that is less subjectively influenced by people and has high reliability of results and self-adaptive model parameters has become a current research trend and difficulty. We followed the methods of Jian and Zhang [4]. Given current knowledge or information, the past (that is, the historical state before the current period) is 
irrelevant for predicting the future (that is, the future state after the current period). In theoretical research, we can use the limit distribution of Markov process as a quantitative indicator of teaching effect; this solves the problem which is due to differences in student foundations that cannot be used to effectively evaluate the quality of teaching by students and can predict the overall learning situation in the future [5].

Therefore, after in-depth study of Markov chain algorithm theory, this research proposed an improved Markov chain teaching quality evaluation model, designed comparative experiments and applied it to the hybrid teaching quality evaluation system of universities, designed a corresponding teaching quality evaluation model, and finally verified its effectiveness through experiments. The mathematical model of mixed classroom teaching quality evaluation given in this research focused on the development and change of the teaching process. For the teaching process that was closely related to the causality of the effect before and after teaching, the model established in this paper was more objective and reasonable for evaluating the quality of teaching.

\section{The Relationship between Markov Chain and Classroom Teaching Quality Evaluation}

There are many phenomena in the real world that have such characteristics: under the condition that the current situation of a certain system is known, the future situation of the system is only related to the present and not directly related to the past history. This phenomenon is called Markov random phenomenon. The mathematical model used to describe this random phenomenon is called the Markov model [6].

\subsection{Introduction to the Basic Theory of Markov Chain.} The Markov chain has "no aftereffect"; that is, to determine the future state of the random process, only the current state is needed, and it has nothing to do with the past state. The stochastic process $\left\{X_{n}, n=0,1,2,3 \ldots \ldots\right\}$ is called a discrete-time Markov chain. If it only takes a finite or listable value, and for any $n \geq 0$, and any state $i, j, i_{0}, i_{1}, \ldots i_{n-1} \in S(\mathrm{~S}$ representative state space), there are

$$
P\left\{X_{n+1}=j\left|X_{n-1}=i_{n-1}, \ldots, X_{1}=i_{1}\right|\right\}=p_{i j} .
$$

In (1), $X_{n-1}=i_{n-1}$ means that the process is in state $i$ at time $n . p_{i j}$ is the one-step transition probability matrix.

The transition probability matrix has, for all $n, m \geq 0, i, j \in S$,

$$
p_{i j}^{m+n}=\sum_{a \in S} p_{i k}^{m} p_{k j}^{n}
$$

The matrix $\left(p_{i j}^{(m)}\right)$ with $p_{i j}^{m}$ as the element is denoted as $X(m)$, which is called the step transition matrix of the Markov chain. It satisfies $X(m+n)=X(m) X(n)$. When $m=l$, it is denoted as $p_{i j}^{(1)}$, the one-step transition matrix is denoted as $p_{i j}$, and then (1) $X^{n}=X(n)=\left(p_{i j}^{(n)}\right)$; (2) when the Markov chain is nonperiodic and irreducible, there is a unique solution under the condition, for example, $X=\left[x_{1}, x_{2}, x_{3}, \ldots x_{m}\right]$.

If a Markov chain is in state $i$ from time $t_{0}$ to time $t_{0+t}$, the transition probability of being in state $j$ is only related to $t$, but to the starting time $t_{0}$ of the transition. Then a Markov chain with this characteristic is called a homogeneous Markov chain. Homogeneous Markov chain has no aftereffect and homogeneity to time can be used as a more reasonable mathematical model of a practical problem in school teaching management [7].

2.2. The Relationship between Markov Chain and Hybrid Classroom Teaching Quality Evaluation. Blended teaching is a new teaching model based on E-learning. It highlights the coordinated and unified teaching process between the teaching in physical places other than the home that can be supervised and the students' online self-controlled learning process. Therefore, online, supervisable physical locations, and integration have become the most critical elements of blended teaching. The blended teaching in this algorithm research is mainly to effectively integrate the advantages of classroom teaching and online teaching. The basic process is embodied in three links: online learning before class, intensive learning in class, and summary learning after class, as shown in Figure 1.

For a certain stage of mixed teaching, although the teaching process has a strong continuity, the most direct impact on the teaching situation of this stage is the teaching situation of the previous stage at this stage; for a long-term continuous teaching process, under the condition that the conditions of teachers, students, and teaching are basically stable, the teaching situation of the whole process tends to be stable. This can be inferred from the changes in the two adjacent stages. It can be seen that the abovementioned law of the mixed-teaching situation is relatively close to the Markov random process, so the Markov chain can be used to construct a mathematical model to evaluate the teaching situation. To describe a system with a mathematical model is to quantify the main indicators in the system and express them in mathematical expressions. According to the common situation in the school's teaching management, this research quantifies the teaching effect index. This is to divide all the teaching objects (experimental class and control class) into $m$ grades for a certain test. For example, it is common to divide the test scores of all students who teach in 5 levels, namely, excellent (90 points or more), good (80-90 points), medium ( $70-79$ points), passing (60-69 points), failing (59 points or less). The purpose of this is to find out the changes in students' test scores after teachers teach. Take the ratio of the number of students in each grade to the total number of students taught by a teacher as a state variable, and use the state probability distribution vector to represent it [8], denoted as

$$
M(t)=x_{1}(t), x_{2}(t), x_{3}(t) \ldots x_{m}(t),
$$

where $t$ is time and $t \in N$. If we try to express $M(t)$ as a homogeneous Markov chain, we can analyse the changing law of the state vector $M(t)$ when $t$ changes. In this way, a 


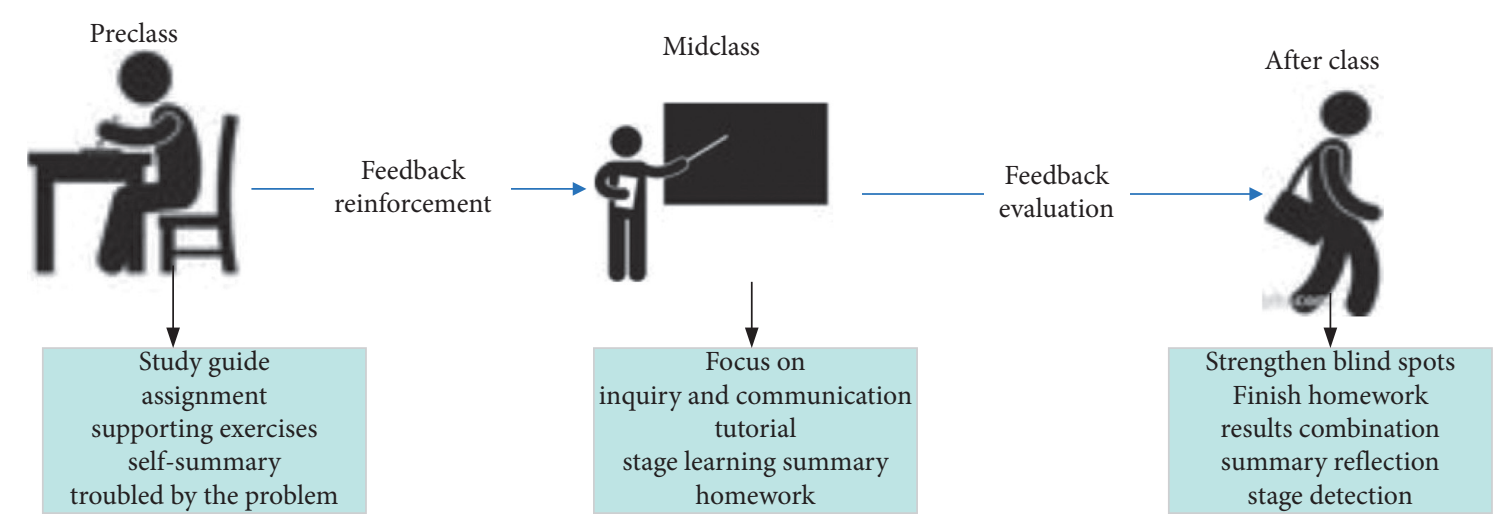

FIgURE 1: The basic process of blended teaching based on Markov algorithm.

mathematical model of teaching quality evaluation can be established by Markov chain's no aftereffect and the homogeneity of time which is not affected by the original difference of the students. First define the initial state vector, denoted as

$$
M(1)=\left(\frac{n_{1}}{n}, \frac{n_{2}}{n}, \cdots, \frac{n_{m}}{n}\right),
$$

where $n$ is the total number of teachers taught by a certain teacher and $n_{a}(a=1,2,3, \cdots m)$ is the number of initial test scores. The initial test score refers to the original score of the student of the course that the teacher holds. With the initial state vector $M(1)$, we can define the transition probability matrix that reflects the transition and change of a teacher's student performance after teaching, denoted as

$$
P=\left(p_{i j}\right)_{m \times m}=\frac{n_{i j}}{n} \text {. }
$$

Here $n_{i j}$ represents the number of students who transfer from the $i$-th grade to the $j$-th grade in the next time (after teaching) relative to the initial grade. The element in the $i$-th row of $\mathrm{P}$ reflects the probability that the student's performance will be transferred to the $j$-th grade of the next test result in the $j$-th grade of the initial grade. Obviously,

$$
M(2)=M(1) P=\left(\sum_{i}^{m} \frac{n_{i 1}}{n}, \sum_{i}^{m} \frac{n_{i 2}}{n}, \sum_{i}^{m} \frac{n_{i 3}}{n}, \ldots, \sum_{i}^{m} \frac{n_{i m}}{n}\right) .
$$

From the above definitions, we can see that $M(t)(t \in N)$ is a homogeneous Markov chain, so we can get

$$
M(t+n)=M(t) P^{n}(n \in N) .
$$

It can be seen from the above that $M(t)$ is an irreducible state and a nonperiodic Markov chain with normal return. Therefore, the limit state distribution of $M(t)$ is the probability distribution of a homogeneous Markov chain in a stationary state. Of course, there are microscopic changes between levels within the steady state system, but the relative number of people at each level no longer changes. According to the ineffectiveness of the Markov chain, the limit state of the system has nothing to do with the initial state of the system; that is, it has nothing to do with $M(1)$, and everything is determined by the transition probability matrix $P$ [9]. Therefore, the transition probability matrix $P$ reflects the teaching quality, teaching conditions, students' mentality (style of study), and social environment. First of all, according to the existing teaching quality evaluation system research and the grading standards of students' student performance, the percentage of passing grades is quantified. The limit state of $M(t)$ shows that when these factors are stable, the overall teaching effect can be achieved under these conditions, and this possible degree has nothing to do with the students' original foundation.

An obvious shortcoming of performance-based evaluation is that it ignores the basic differences of students, and the students' learning progress from the early stage to the later stage is not reflected. Therefore, grades alone cannot truly reflect the quality of teaching at this stage. The evaluation method established by the Markov chain can make up for this defect [10-12]. The specific method is as follows.

On the basis of the previous algorithm, a "progress" model is established. After the stage of teaching, the original $i$-level becomes $j$-level. When $i \geq j$, it is progress, and when $i \leq j$, it is regress. Let

$$
w_{i j}=(i-j) p_{i j}=(i-j)^{3} \frac{n_{i m}}{n_{i}},(i=1,2,3, \ldots, q ; j=1,2,3, \ldots, q ;) .
$$

Among them, $w_{i j}$ is called the degree of transfer progress of $p_{i j}$, and $(i-j)^{3}$ is called the weight of $p_{i j}$. The magnitude and sign of the value of $(i-j)$ indicate the degree of progress or regression. The index " 3 " is used to adjust the plus and minus and the weight. The matrix $w_{i j}$ with $W=\left(w_{i j}\right)_{q^{2}}=(i-j)^{3} n_{i m} / n_{i q^{2}}$ as the element is called the progress matrix of the transition matrix A; $E(w)=\sum_{i=1}^{q}$ $\sum_{j=1}^{q} w_{i j}=\sum_{i=1}^{q}(i-j)^{3} \sum_{j=1}^{q} p_{i j}=\sum_{i=1}^{q}(i-j)^{3} \sum_{j=1}^{q} n_{i m} / n_{i}$ is called the efficiency of transition matrix $p_{i j}$. $E(w)$ is used as a numerical feature to evaluate the quality of teaching. The larger $E(w)$, the more the transformations belonging to $i \geq j$, and the higher the quality of teaching.

At the same time, the long-term prediction of teaching is of great significance in teaching. Throughout the teaching 
process, although the frequency of students at all levels in each stage of the examination is constantly changing, the transfer ratio of students at all levels tends to be stable. Each stage can be approximately regarded as having the same one-step transition matrix, the entire teaching process is a Markov chain, and this Markov chain is nonperiodic and irreducible [13-15]. We can use the ergodic theorem of the Markov chain to establish a mathematical model for teaching long-term prediction. The specific method is as follows.

Construct the state vector $P$ and the one-step transition matrix $A$ according to the test scores of the last two stages, solving the system of equations $X=X A$, where $\sum_{i=1}^{q} x_{i}=1$ is the limit vector of the transition matrix A (i.e., the solution of the system of equations): $Y=\left|p_{1}, p_{2}, p_{3}, \ldots p_{q}\right|$. According to the principle of maximum probability, $\max \left\{p_{1}, p_{2}, p_{3}, \ldots p_{q}\right\}$ is taken as the grade evaluation of the long-term effect of teaching. If the overall situation is evaluated, a comprehensive score can be further calculated for evaluation.

\section{The Improvement of Markov Chain in the Evaluation of Mixed Classroom Teaching Quality}

The hybrid teaching method is formed by applying new technical tools to traditional teaching practice with the advancement of information technology, and making a deep analysis of its existing problems [16-19]. In the actual application process, the blended teaching mode shows several obvious advantages over traditional teaching methods. The details are shown in Figure 2.

As mentioned earlier, in the practice of mixed classroom teaching, the Markov chain method is used for evaluation. Generally, the relationship between two successive value states (such as two test results) is used to describe the transition probability matrix [20, 21]. And from this, the practice of the evaluation object to reach the current state is evaluated. However, whether the value scales used in the previous two times are the same and whether the scenes evaluated on the evaluation object are the same two times will affect the determined state matrix. Then according to the application of Markov chain transition probability matrix, the traditional teaching and flipped classroom teaching are compared, and whether the effectiveness of flipped classroom teaching quality has changed significantly is studied $[22,23]$. When using the obtained probability vector to solve the system of equations, the characteristic root $\lambda$ of the vector has been artificially determined to be 1 , and then the evaluation standard is established. As for whether each state is stable, the user should verify it by solving. In actual teaching applications, the evaluator does not consider this condition but assumes that it reaches a steady state. In fact, this condition has a certain impact on the construction of a stable probability distribution. Therefore, it is necessary to improve the algorithm of Markov chain in the evaluation of mixed classroom teaching quality.

Combine the student's two exam results into a sample $\left(f_{11}, f_{12}, f_{13}, \cdots f_{1 m} ; f_{21}, f_{22}, f_{23}, \cdots f_{2 m}\right)$, and find the average $(\bar{F})$ and standard deviation $(s)$. Studies have shown that students' academic performance is basically normally distributed or close to a normal distribution. Therefore, according to the law of normal distribution, $(\bar{F})$ and $s$ can be used to divide $m$ levels within a certain interval. Then calculate the state vector of the ratio of the number of students in each level to the total number of students in the previous exam by $m$ levels, denoted by $A$ :

$$
A=\left(n_{1} / n, n_{2} / n, n_{3} / n, \ldots n_{q} / n\right)
$$

where $n$ is the total number of students and $q$ is the number of $i=1,2,3, \ldots q$-level students. A represents the set of these vectors, from the first to $n$. Among them, each level represents the weight of each individual student in total.

In order to examine the teaching effect, it is necessary to analyse the changes in each level of the abovementioned students in the second examination. Similarly, the scores of the second test are also calculated according to the interval standards of $m$ levels, and the frequency of students included in each level is counted, so as to find the transition matrix $P$ in the next step:

$$
P=\left[\begin{array}{cccc}
\frac{n_{11}}{n_{1}} & \frac{n_{12}}{n_{1}} & \cdots & \frac{n_{1 q}}{n_{1}} \\
\frac{n_{21}}{n_{2}} & \frac{n_{22}}{n_{2}} & \cdots & \frac{n_{2 q}}{n_{2}} \\
\frac{n_{31}}{n_{3}} & \frac{n_{31}}{n_{3}} & \cdots & \frac{n_{3 q}}{n_{3}} \\
\vdots & \vdots & \cdots & \vdots \\
\frac{n_{q 1}}{n_{q}} & \frac{n_{q 2}}{n_{q}} & \cdots & \frac{n_{q q}}{n_{q}}
\end{array}\right]=p_{i j} q^{2} .
$$

Among them, $n_{i}$ still represents the number of students in the $i$-th grade at the initial stage, and $n_{i j}$ represents the number of students who belong to the $i$-th grade after the stage of teaching, and their scores belong to the number of students in the $j$-th grade and satisfy $\sum_{j=1}^{q} p_{i j}=1, i, j$ $=1,2,3, \cdots q$. This not only solves the problem of inconsistent value states but also gives full play to the characteristics of the one-step transfer matrix of the Markov chain. That is to say, it can eliminate the basic differences but also reflect the advantages of its change efficiency $[24,25]$.

3.1. Establishing a Mixed Classroom Teaching Model and Analysis. In order to avoid the multistep transition of the Markov chain, and the demanding condition of stable probability distribution that can only be obtained in the limit state, the student's "progress" is used to consider whether the student is progressing or regressing. The detailed analysis of time benefit analysis is based on two tests. That is, "pretest results and posttest results." Now suppose that cultivating $i$ - 


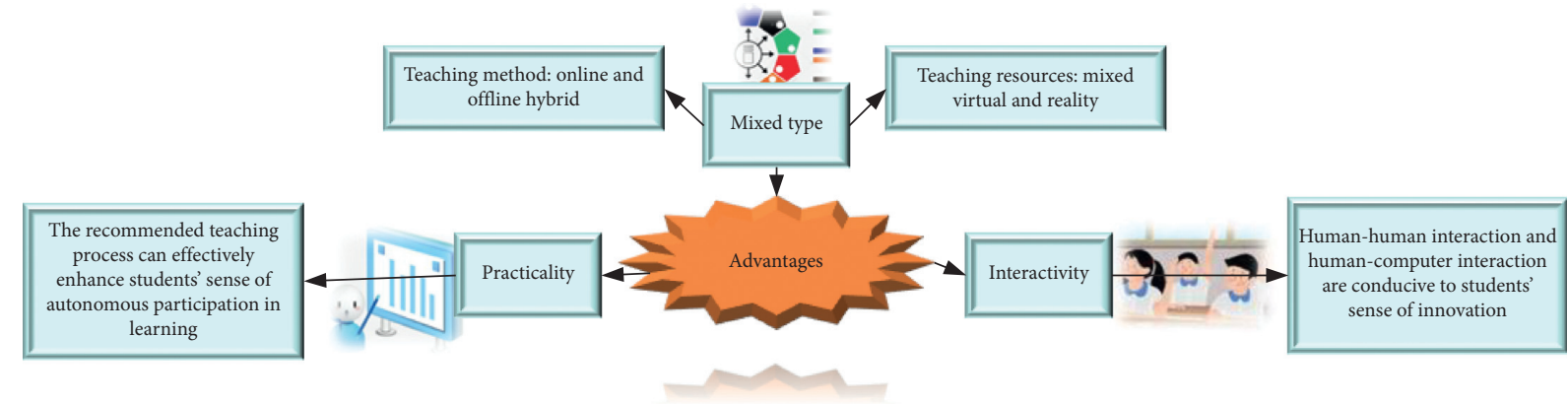

FIgURE 2: The advantages of a blended teaching model.

level students to $j(i \geq j)$ level students is progress, and cultivating $i$-level students to $j(i \leq j)$ level students is regression. Grasping this point can eliminate basic differences and at the same time reflect the effectiveness of teaching. In order to accurately extract the change information from the transition matrix, the following algorithm model is established: $W=\left(W_{i j}\right)_{q^{2}}=\left((i-j)^{3} / n_{i}\right) q^{2}$ is called the progress matrix of the transition matrix $p_{i j}$. $E(w)=\sum_{i=1}^{q} \sum_{j=1}^{q} W_{i j}=$ $\sum_{i=1}^{q} \sum_{j=1}^{q}(i-j)^{3} p_{i j}\left(n_{i j} / n_{i}\right)$ is called the efficiency of transition matrix $p_{i j}$.

\section{Algorithm Analysis of Mixed Classroom Teaching Quality Evaluation Based on the Improved Markov Chain}

Considering the unique characteristics of blended teaching compared with traditional teaching methods, certain basic principles should be followed in the process of constructing a blended teaching quality evaluation system, as shown in Figure 3.

This part of the content is based on a total of 450 students in three grades of 2017, 2018, and 2019 (150 students for each grade) of a university. Each grade selects students from Guangdong in the five majors of information management, basic physics, medical laboratory, pharmacy, and life sciences. This research material mainly selects the college English test scores of the three grade students in the previous year and the current year as the research objects. By using the Markov chain statistical model to analyse the transition probability and limit distribution of the grades of students in each grade, the teaching effect and quality of each grade are analysed.

\subsection{Construction of a Hybrid Teaching Quality Evaluation} System Model Based on the Markov Chain Algorithm. Combining the characteristics of the mixed-teaching model and following the basic principles of the construction of the mixed-teaching quality evaluation index system, we have constructed an evaluation model including basic evaluation, process evaluation, and result evaluation (Figure 4). Through careful analysis of the changes of the students' two tests in different grades, the transfer of student group performance in the two tests is studied, and a transfer matrix is formed to give the expected time for students of each level to reach the predetermined goal.
4.2. Determination of the Set of Evaluation Factors and Establishment of the Set of Evaluation Indicators and Comments. By consulting the literature and combining the characteristics of college English teaching courses, summarize the classroom teaching quality evaluation factors shown in Figure 5. To describe a system with a mathematical model is to quantify the main indicators in the system and express them in mathematical expressions. According to the content mentioned above, the comment set of this article = "excellent," "good," "medium," "qualified," and "unqualified". The corresponding comment score set $=$ excellent (90 points or more), good (80-90 points), medium (70-79 points), qualified (60-69 points), and failing (59 points or less).

4.3. Determination of the Index Weight. When calculating the weight of the influence factor applied to the evaluation of teaching quality, the principle of Markov chain algorithm is used to input the relative importance of the evaluation index of teaching attitude, the evaluation index of teaching content, the evaluation index of teaching method, and the evaluation index of teaching, respectively. According to the common situation in school teaching management, this study quantifies the teaching effect index, which is to divide all the teaching objects (experimental class and control class) into $m$ grades for a certain test, that is, as the judgment matrix of the teaching quality evaluation system, the data required for calculation in the teaching quality evaluation factor of the first-level criterion level. Specifically, the process of calculating the weights of the teaching quality evaluation factors at the first-level criterion level and the second-level criterion level is shown in Figure 6.

Thirty representative teachers in the frontline and teaching management positions were invited to fill in the Questionnaire on the Importance of Teaching Quality Evaluation Indexes in Colleges and Universities, and 15 high-quality questionnaires were selected as the survey results. According to the results of the questionnaire, the weight of each evaluation index is calculated. Table 1 shows the weights of evaluation indicators calculated based on the results of a questionnaire. In order to ensure correctness and operation efficiency, this article uses Python programming to achieve classroom teaching quality evaluation, and the efficiency has been very well improved. After the weights of the 15 experts' evaluation indicators are calculated, and then the arithmetic average value is calculated, the final weight value of each indicator can be obtained. 


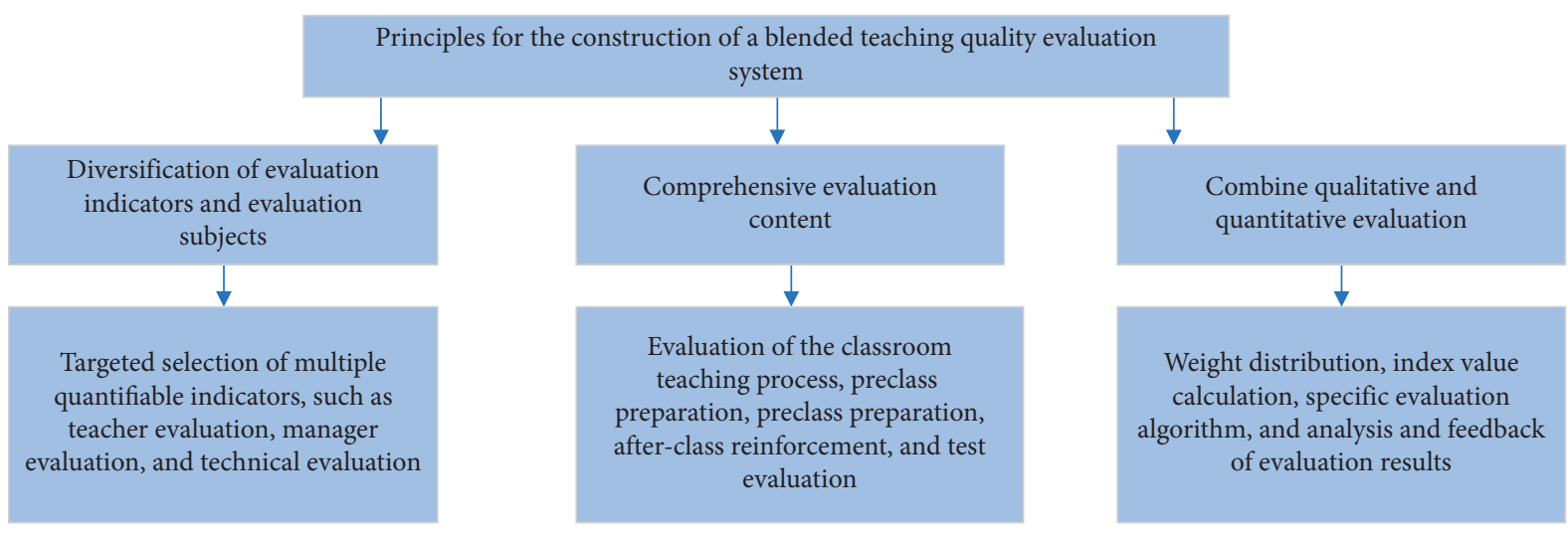

Figure 3: Principles that should be followed to construct a blended teaching quality evaluation system.

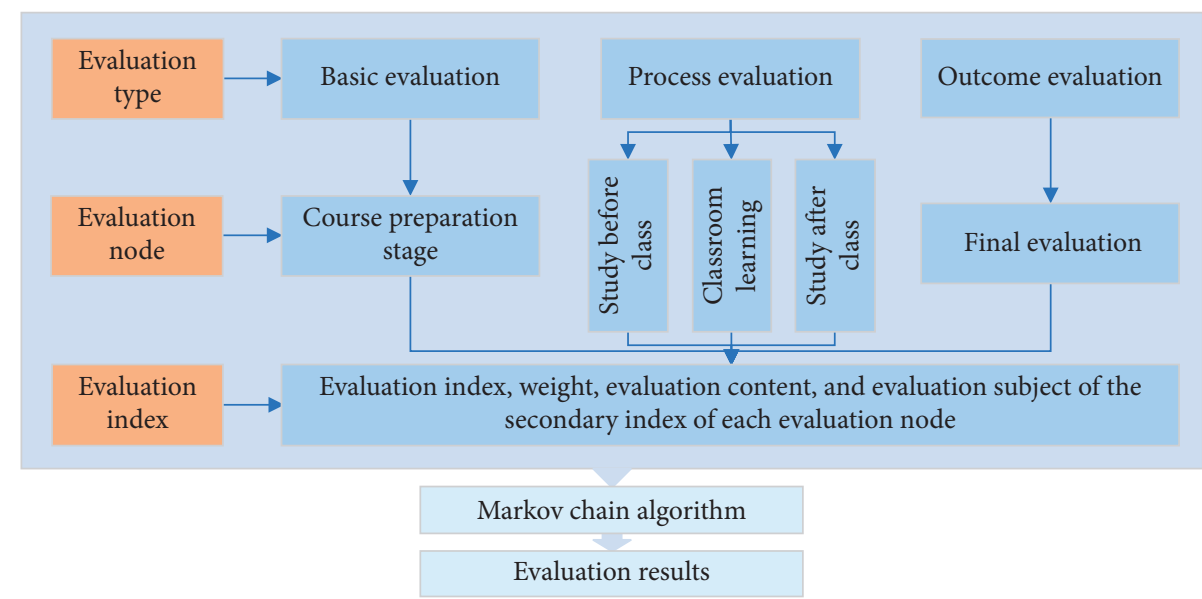

Figure 4: Hybrid teaching quality evaluation system model based on Markov chain algorithm.

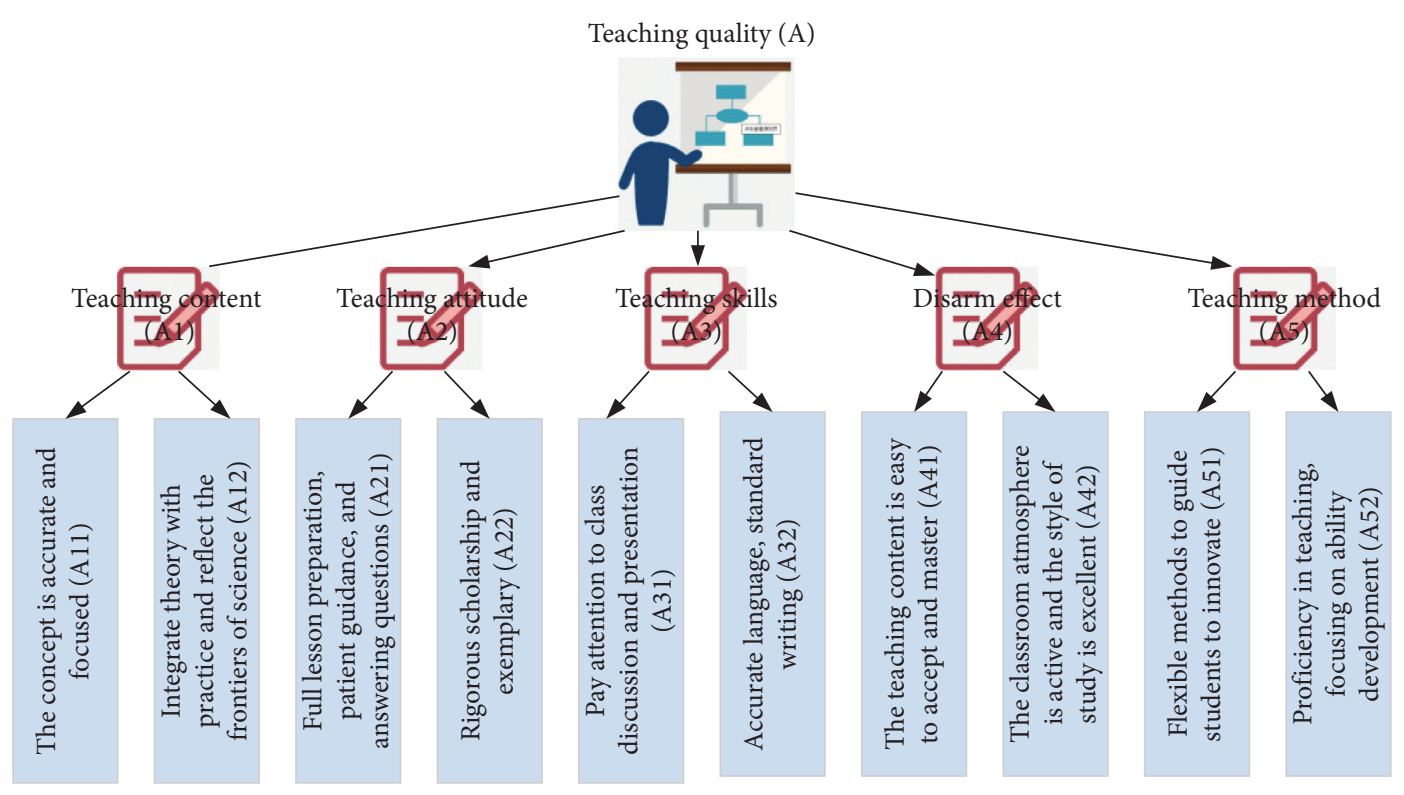

FIGURE 5: Analysis of evaluation factors of classroom teaching quality. 


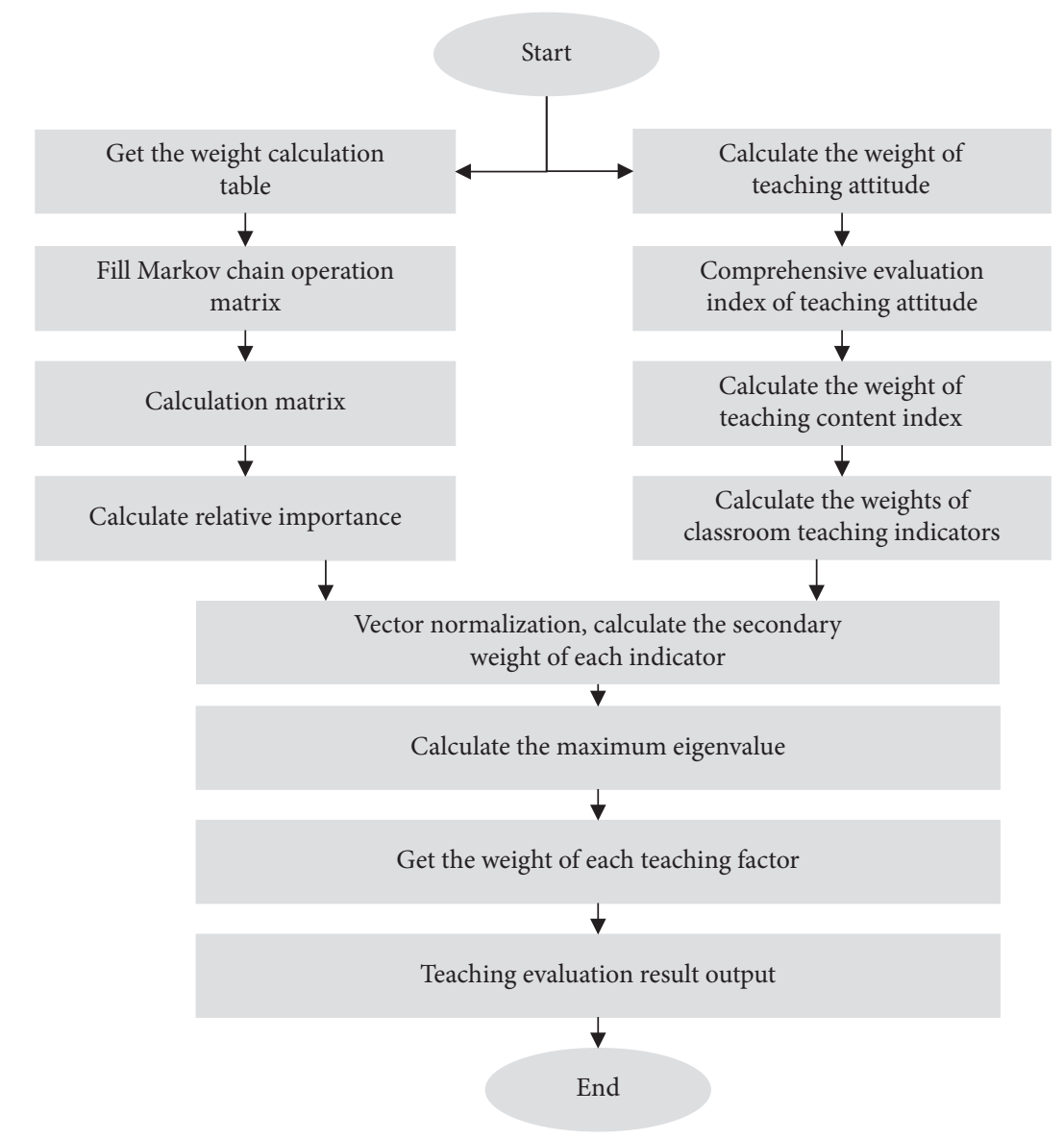

FIGURE 6: The process of determining the weights of teaching quality evaluation indicators.

TABLE 1: Weights of evaluation indicators calculated based on the results of a questionnaire.

\begin{tabular}{|c|c|c|c|c|}
\hline Target layer & \multicolumn{2}{|c|}{ Criterion layer } & \multirow{2}{*}{$\begin{array}{c}\text { Index layer } \\
\text { A11 }(0.3333)\end{array}$} & \multirow{2}{*}{$\begin{array}{c}\text { Comprehensive weight } \\
0.0466\end{array}$} \\
\hline \multirow{10}{*}{ Teaching quality (A) } & \multirow{2}{*}{ Teaching content (A1) } & \multirow{2}{*}{0.1594} & & \\
\hline & & & A12 (0.6667) & 0.0634 \\
\hline & \multirow{2}{*}{ Teaching attitude (A2) } & \multirow{2}{*}{0.1011} & A21 (0.6667) & 0.0543 \\
\hline & & & $\mathrm{A} 22(0.3333)$ & 0.0124 \\
\hline & \multirow{2}{*}{ Teaching skills (A3) } & \multirow{2}{*}{0.1916} & A31 (0.6667) & 0.0671 \\
\hline & & & A32 (0.3333) & 0.1057 \\
\hline & \multirow{2}{*}{ Disarm effect (A4) } & \multirow{2}{*}{0.3364} & A41 (0.5000) & 0.2581 \\
\hline & & & $\mathrm{A} 42(0.5000)$ & 0.1647 \\
\hline & \multirow{2}{*}{ Teaching method (A5) } & \multirow{2}{*}{0.2547} & A51 (0.3333) & 0.1014 \\
\hline & & & A52 (0.6667) & 0.0860 \\
\hline
\end{tabular}

4.4. Collection and Arrangement of Teaching Quality Data. Choose 5 classes (30 students in each class) with a balanced level of learning ability in the same major and taught by the same experienced teacher with a higher level of teaching. In each class, the first stage of teaching uses traditional teaching methods, and the second stage of teaching uses a mixed classroom teaching model. On the basis of the analysis of the student population, we use SPASS statistical software and MATLAB mathematics software for data processing, which can obtain information on the teaching quality and effectiveness of the two classes. Two assessments are carried out during the teaching process, the spot midterm test and the final exam are combined, and the final scores are all recorded using the average score. The traditional teaching assessment score data is shown in Figure 7.

Through the second stage of the mixed classroom teaching study, the class students' academic records of each score segment of the two test scores are sorted, and the average conversion rate of each score segment is $p_{i j}, i=1,2,3, \ldots 10 ; j=1,2,3, \ldots 10 . i=1$ represents the average percentage of students' academic performance in the traditional teaching $[0,10]$ fractional period; $j=1$ represents the average percentage of students' academic performance in 


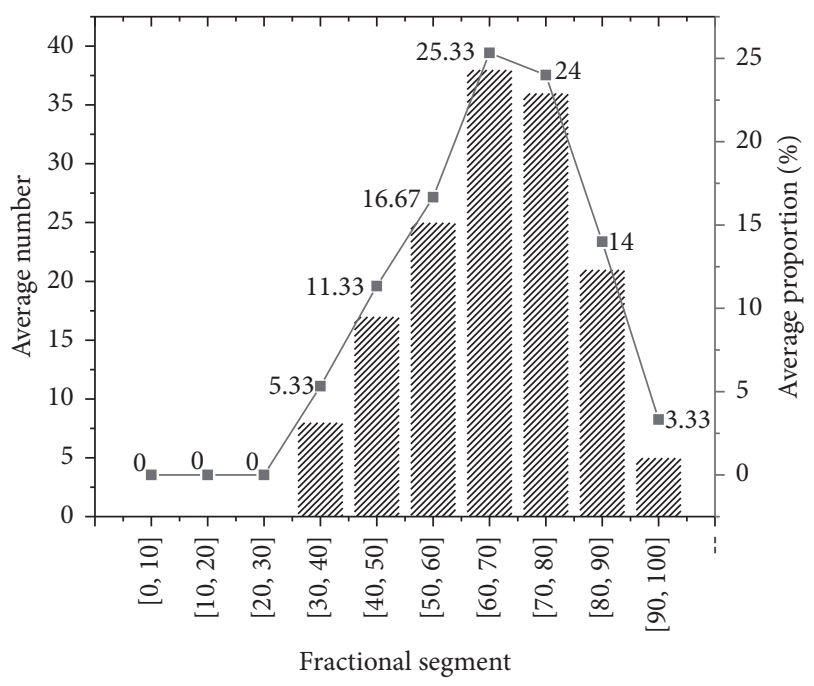

FIgUre 7: Performance data of traditional teaching.

the $[0,10]$ fractional segment under the mixed-teaching classroom teaching mode, so on and so forth. The purpose of this is to find out the changes in the students' test scores after the teacher teaches. This level change is a discrete state change, so the consideration is more in line with objective reality. The specific transition probability is

$$
P=\left[\begin{array}{cccccccc}
0.87 & 0.06 & 0.03 & 0.01 & 0 & 0 & 0 & 0 \\
0.02 & 0.82 & 0.07 & 0.01 & 0 & 0 & 0 & 0 \\
0 & 0 & 0.09 & 0.08 & 0.01 & 0 & 0 & 0 \\
0 & 0 & 0.02 & 0.01 & 0.01 & 0 & 0 & 0 \\
0 & 0 & 0 & 0.05 & 0.01 & 0 & 0 & 0 \\
0 & 0 & 0 & 0 & 0.01 & 0.01 & 0.08 & 0 \\
0 & 0 & 0 & 0 & 0.15 & 0.02 & 0.86 & 0 \\
0 & 0 & 0 & 0 & 0.02 & 0.01 & 0.15 & 0.07 \\
0 & 0 & 0 & 0 & 0 & 0.01 & 0.94 & 0.08 \\
0 & 0 & 0 & 0 & 0 & 0.01 & 0.03 & 0.95
\end{array}\right] .
$$

\section{Result Analysis of Classroom Teaching Quality}

5.1. Markov Chain Algorithm Analysis of the College English Test Scores of the Previous Year and the Current Year. According to the average scores of the 2017, 2018, and 2019 exams in Table 2, the teaching effect is considered. The 2017 grade is the highest, but this kind of teaching effect evaluation method ignores the students' original foundation. Therefore, it is necessary to analyse the teaching effect of different grades through the Markov chain model. The solution of this set of equations gives the expected time for students of each level to reach the predetermined goal. See Table 2 for the status transition of the three grades from the previous year to the current year. The English score transfer matrices of the three grades obtained from Table 2are

$$
\begin{aligned}
& W_{\text {Class of } 2017}=\left[\begin{array}{cccc}
0 & 0 & 0 & 0 \\
\frac{5}{20} & \frac{13}{20} & \frac{2}{20} & 0 \\
\frac{11}{141} & \frac{98}{141} & \frac{5}{141} & 0 \\
\frac{3}{136} & \frac{62}{136} & \frac{8}{136} & 0 \\
0 & \frac{3}{35} & \frac{16}{35} & 0
\end{array}\right] \text {, } \\
& W_{\text {Class of } 2018}=\left[\begin{array}{cccc}
0 & 0 & 0 & 0 \\
\frac{52}{41} & \frac{16}{41} & 0 & 0 \\
\frac{15}{126} & \frac{84}{126} & \frac{8}{126} & \frac{1}{126} \\
\frac{4}{151} & \frac{51}{151} & \frac{17}{151} & 0 \\
0 & \frac{8}{68} & \frac{13}{68} & 0
\end{array}\right] \text {, } \\
& W_{\text {Class of } 2019}=\left[\begin{array}{cccc}
0 & 0 & 0 & 0 \\
0 & \frac{16}{41} & 0 & 0 \\
\frac{18}{119} & \frac{26}{119} & \frac{7}{119} & 0 \\
\frac{8}{184} & \frac{47}{184} & \frac{22}{184} & 0 \\
0 & \frac{9}{41} & \frac{21}{41} & 0
\end{array}\right] .
\end{aligned}
$$

According to the linear equations, the distribution vector of 2017 students' English scores is

$W_{\text {Grade 2017 }}=(0.1264,0.6154,0.0029,0.1546,0.1325)$.

The distribution vector of English scores of 2018 students is

$W_{\text {Grade 2018 }}=(0.1514,0.5428,0.0526,0.1479,0.1132)$.

The score distribution vector for the 2019 grade is

$W_{\text {Grade 2019 }}=(0.0121,0.7814,0.1019,0.1121,0.0124)$. 
TABLE 2: The status of the transition from the previous year to the current year's English scores in Grades 2017, 2018 , and 2019.

\begin{tabular}{|c|c|c|c|c|c|c|c|c|}
\hline & & \multicolumn{7}{|c|}{ English test scores of the year } \\
\hline & & & $\mathrm{n} 1$ & $\mathrm{n} 2$ & n3 & $\mathrm{n} 4$ & n5 & Total \\
\hline \multirow{6}{*}{ Grade 2017} & \multirow{6}{*}{ English test results of the previous year } & n1 & 0 & 0 & 0 & 0 & 0 & 0 \\
\hline & & $\mathrm{n} 2$ & 1 & 6 & 7 & 8 & 0 & 22 \\
\hline & & n3 & 7 & 9 & 16 & 10 & 0 & 42 \\
\hline & & $\mathrm{n} 4$ & 2 & 15 & 25 & 2 & 0 & 44 \\
\hline & & n5 & 1 & 19 & 9 & 13 & 0 & 42 \\
\hline & & Total & 11 & 49 & 57 & 33 & 0 & 150 \\
\hline \multirow{6}{*}{ Grade 2018} & \multirow{6}{*}{ English test results of the previous year } & $\mathrm{n} 1$ & 0 & 0 & 0 & 0 & 0 & 0 \\
\hline & & $\mathrm{n} 2$ & 2 & 8 & 7 & 8 & 0 & 25 \\
\hline & & $\mathrm{n} 3$ & 6 & 1 & 14 & 10 & 0 & 31 \\
\hline & & $\mathrm{n} 4$ & 5 & 19 & 18 & 8 & 1 & 51 \\
\hline & & $\mathrm{n} 5$ & 0 & 10 & 15 & 18 & 0 & 43 \\
\hline & & Total & 13 & 38 & 54 & 44 & 1 & 150 \\
\hline \multirow{6}{*}{ Grade 2019} & \multirow{6}{*}{ English test results of the previous year } & $\mathrm{n} 1$ & 0 & 0 & 0 & 0 & 0 & 0 \\
\hline & & $\mathrm{n} 2$ & 2 & 6 & 3 & 8 & 0 & 19 \\
\hline & & n3 & 5 & 9 & 10 & 1 & 0 & 25 \\
\hline & & $\mathrm{n} 4$ & 1 & 20 & 9 & 17 & 0 & 47 \\
\hline & & $\mathrm{n} 5$ & 0 & 25 & 21 & 13 & 0 & 59 \\
\hline & & Total & 8 & 60 & 43 & 39 & 0 & 150 \\
\hline
\end{tabular}

It can be seen from the above vector distribution that the failing rate of 2017 students is $13.25 \%$, the failing rate of 2018 students is $11.32 \%$, and the failing rate of 2019 students is $0.12 \%$. Judging from the failing rate, the students in the class of 2019 are the best overall. In order to quantify the teaching effect, the four grades were assigned scores: excellent $=90$, $\operatorname{good}=80$, medium $=70$, pass $=60$, and failing $=50$. Using the limit distribution to perform weighted average, the quantified values are as follows:

$$
\begin{aligned}
& W_{\text {Grade 2017 }}=(0.1264 \times 90+0.6154 \times 80+0.0029 \times 70+0.1546 \times 60+0.1325 \times 50)=76.712, \\
& W_{\text {Grade 2018 }}=(0.1514 \times 90+0.5428 \times 80+0.0526 \times 70+0.1479 \times 60+0.1132 \times 50)=75.374, \\
& W_{\text {Grade 2019 }}=(0.0121 \times 90+0.7814 \times 80+0.1019 \times 70+0.1121 \times 60+0.0124 \times 50)=78.080 .
\end{aligned}
$$

The above amount is in the three grades, although the students' English test scores of the previous year and the current year are the highest in the 2017 grade. But the Markov chain model shows that the 2019 students have made the most progress and the teaching effect is the best. And there is no significant difference in the overall progress of the three grades. This also shows that the school's English teaching level has improved in 2019.

5.2. Evaluation and Analysis of Teachers' Teaching Work. Taking the mixed teaching of college English teaching in the two classes (Class 1 and Class 2) of the university's information management major in Grade 2018 as an example, the improved Markov algorithm is used to evaluate the teaching work of two college English teachers. Any kind of mathematical quality index of each person corresponds to a degree of high, medium, and low grade, which is determined by the attribute measurement function of the index. The performance statistics and transfer of the two classes are shown in Table 3.
From Table 3, we can see that the transition probability matrices of the college English test scores of the two classes are

$$
P_{1}=\left[\begin{array}{ccccc}
\frac{4}{7} & \frac{1}{7} & 0 & 0 & 0 \\
\frac{5}{30} & \frac{11}{30} & \frac{21}{30} & 0 & 0 \\
0 & \frac{6}{46} & \frac{8}{46} & \frac{11}{46} & \frac{4}{46} \\
0 & \frac{1}{17} & \frac{7}{17} & \frac{1}{17} & \frac{9}{17} \\
0 & 0 & \frac{1}{8} & \frac{4}{8} & \frac{3}{8}
\end{array}\right], P_{2}=\left[\begin{array}{ccccc}
\frac{5}{13} & \frac{1}{13} & \frac{11}{13} & 0 & 0 \\
\frac{4}{41} & \frac{13}{41} & \frac{19}{41} & \frac{15}{41} & 0 \\
0 & \frac{6}{9} & \frac{1}{9} & \frac{7}{9} & \frac{2}{9} \\
0 & \frac{13}{18} & \frac{6}{18} & \frac{11}{18} & \frac{2}{18} \\
0 & \frac{1}{8} & \frac{1}{8} & \frac{4}{8} & \frac{3}{8}
\end{array}\right]
$$


TABLE 3: The transfer situation of college English teaching grades in Grade 2018 of information management.

\begin{tabular}{lccccccccc}
\hline & & Class 1 & & \multicolumn{4}{c}{ Class 2 } \\
& $\mathrm{n} 1$ & $\mathrm{n} 2$ & $\mathrm{n} 3$ & $\mathrm{n} 4$ & $\mathrm{n} 5$ & $\mathrm{n} 1$ & $\mathrm{n} 2$ & $\mathrm{n} 3$ & $\mathrm{n} 4$ \\
\hline $\mathrm{n} 1$ & 0 & 0 & 0 & 0 & 0 & 0 & 0 & 0 & 0 \\
$\mathrm{n} 2$ & 0 & 4 & 1 & 2 & 0 & 0 & 1 & 0 & 2 \\
$\mathrm{n} 3$ & 1 & 1 & 5 & 2 & 0 & 0 & 5 & 2 & 0 \\
$\mathrm{n} 4$ & 2 & 7 & 1 & 1 & 1 & 3 & 5 & 2 & 2 \\
$\mathrm{n} 5$ & 0 & 0 & 0 & 2 & 0 & 0 & 0 & 0 & 0 \\
Total & 3 & 12 & 7 & 7 & 1 & 3 & 11 & 4 & 12 \\
\hline
\end{tabular}

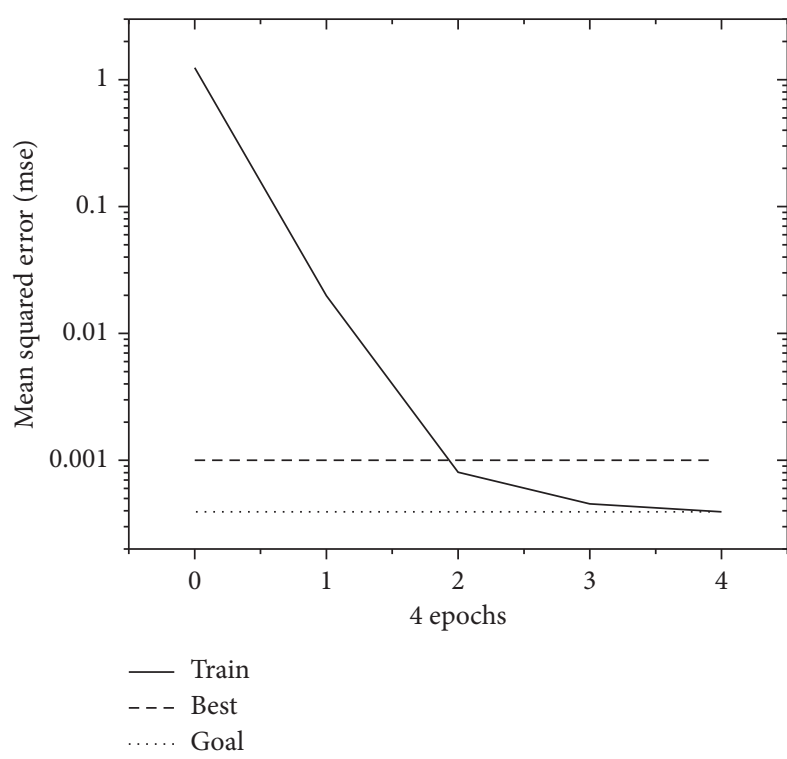

(a)

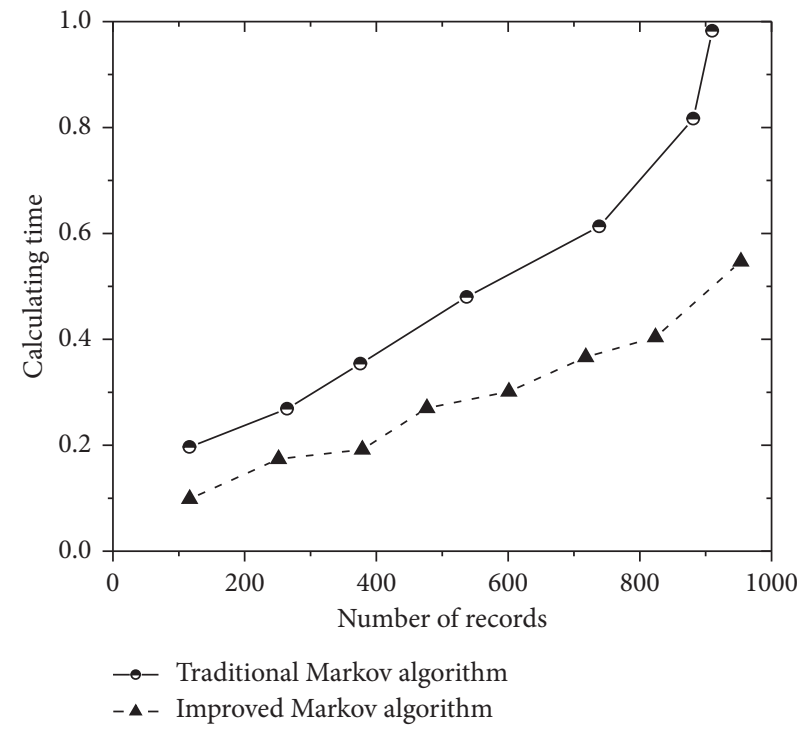

(b)

Figure 8: Network training error curve obtained after 4 iterations (a) and algorithm time comparison chart before and after improvement (b).

Using Formula (3) to find the stable distribution of the transfer of the college English test scores of the two classes, the equations can be listed:

$$
\left\{\begin{array}{l}
\beta=P^{T} \beta, \\
\sum_{j=1}^{w} \beta_{j}=1 .
\end{array}\right.
$$

Putting P1 and P2 into (3) can be solved:

$$
\begin{aligned}
& \beta_{1}=\left(\begin{array}{lllll}
0.21 & 0.35 & 0.27 & 0.16 & 0.07
\end{array}\right)^{T}, \\
& \beta_{2}=\left(\begin{array}{lllll}
0.24 & 0.35 & 0.35 & 0.05 & 0.04
\end{array}\right)^{T} .
\end{aligned}
$$

Finally, using Formula (5), the scores of the quantitative index of epidemiology teaching effect in two classes can be obtained: $W_{1}=g \beta_{1}=71.7 ; W_{2}=g \beta_{2}=73.5$. It can be seen that $W_{1} \leq W_{2}$. The above method of solving the expected time is a simple method, but it loses a lot of important and useful information. So we can introduce a matrix and use the method of solving the inverse matrix to get a lot of gains. This shows that, after excluding the influence of the different foundations of the two classes, the teaching effect of college English in Class 2 is better than that in Class 1.
5.3. Algorithm Training of the Network and Algorithm Time Comparison of the Improved Markov Chain Algorithm. This article takes a college English teaching supervision expert evaluation of the first semester of the 2017-2018 academic year as an example. We measure each indicator in the form of usual tests, investigations, and questionnaires, obtain the percentage of each indicator, and divide these scores into three grades: high, medium, and low. The evaluation indicators in this research are used as the input sample of the network, and one evaluation result is used as the output of the network. According to the Markov algorithm formula, train these data and set the network parameters in the MATLAB simulation program: the number of network layers is 2, the transfer function of the hidden layer is logsig (s-type logarithmic function), the transfer function of the output layer is purelin (linear function), and the training target is 0.002 . Finally, it is determined that the number of hidden layer nodes with the smallest mean square error is 9. At this time, after 4 iterations, the network training error curve is shown in Figure 8(a).

It can be seen from Figure 8(a)that the algorithm of this study can meet the accuracy requirements through 4 iterations, the training time is short, and the training results meet the desired goals. 
The improved Markov chain algorithm reduces the generation of new duplicates during the union, thus improving the operating efficiency. We stipulate that an attribute measurement function is determined by the performance distribution of each index and the membership degree of the research question. The time comparison between the improved algorithm in this paper and the classic algorithm is shown in Figure 8(b). From Figure 8(b), it can be seen that the running time of the improved algorithm is generally half the time of the traditional algorithm. The improved algorithm reduces the access to the database, so the time increase is relatively slow.

\section{Conclusion}

The Markov chain model has a wide range of applicability. The evaluation object can be a university, a teacher, a class, or a certain student. It can also be used to evaluate the quality of teaching materials, students' abilities (character, interest, physical fitness, etc.), test paper quality, etc. For simple systems, single-level evaluation can be used, and for complex systems, multilevel evaluation can be used, but the mathematical methods are the same. The teaching quality evaluation based on Markov chain random process theory is a dynamic evaluation method based on development. In the case of excluding external factors, make full use of the large amount of information contained in the one-step transition probability matrix of the Markov chain to evaluate the changing law of teachers' teaching quality. Based on the theory of Markov chain, this paper establishes an evaluation index system for teachers' teaching quality in Gao School through the analysis of a large number of documents and determines the index weights. Then a fuzzy dynamic comprehensive evaluation model of university teachers' teaching quality based on Markov chain is established. And based on the evaluation data of the students in the teaching class of a certain main course teacher in a certain university, the dynamic evaluation model established in this paper is used to evaluate the teaching quality. The evaluation results show that the model has high scientificity and practicability. The dynamic evaluation model is not only based on the first evaluation behaviour of students but also can continuously evaluate the teaching quality of current and future teachers. It can also find out the problems in the teaching process of teachers in time and solve them in time to achieve the expected training goals and improve the teaching level and the purpose of talent training.

\section{Data Availability}

The data used to support the findings of this study are available from the corresponding author upon request.

\section{Conflicts of Interest}

The author declares no conflicts of interest.

\section{Acknowledgments}

The author acknowledges the Key Projects of the Ministry of Education in 2016 of the 13th Five-Year Plan of National Education Science: Research on the Application of Visual Analysis Technology for the Professional Development of University Teachers (no. DCA160264).

\section{References}

[1] Q. Jian, "Multimedia teaching quality evaluation system in colleges based on genetic algorithm and social computing approach," IEEE Access, vol. 7, pp. 90-99, 2019.

[2] W. Xuhui and X. Jian, "The model of teaching quality evaluation based on BP neural networks and its application," in Proceedings of the 2009 First International Workshop on Education Technology and Computer Science, pp. 916-919, Wuhan, China, March 2009.

[3] M. Lianguang, "Evaluation of the teaching effect of construction engineering cost software based on the Markov chain," in Proceedings of the 2012 Second International Conference on Intelligent System Design and Engineering Application, pp. 832-836, Sanya, China, January 2012.

[4] W. Jian and W. Zhang, "Fuzzy mathematics and machine learning algorithms application in educational quality evaluation model," Journal of Intelligent and Fuzzy Systems, vol. 39, no. 4, pp. 5583-5593, 2020.

[5] X. Y. Huang and S. Q. Feng, "Research on the teaching quality evaluation for the physical education in colleges based on the ahptopsis," Chemical Engineering Transactions, vol. 46, pp. 487-492, 2015.

[6] M. Luo, "Application of AHP-DEA-FCE model in college English teaching quality evaluation," International Journal of Applied Mathematics and Statistics, vol. 51, no. 21, pp. 101108, 2013.

[7] X. F. Zhao, "TOPSIS method for interval-valued intuitionistic fuzzy multiple attribute decision making and its application to teaching quality evaluation," Journal of Intelligent and Fuzzy Systems, vol. 26, no. 6, pp. 49-55, 2014.

[8] C. H. Mao, "Research on information system for teaching quality evaluation model of business English translation based on SVM," Advanced Materials Research, vol. 886, pp. 552555, 2014.

[9] Y. S. Zeng, "Evaluation of physical education teaching quality in colleges based on the hybrid technology of data mining and hidden Markov model," International Journal of Emerging Technologies in Learning (IJET), vol. 15, no. 1, pp. 4-15, 2020.

[10] Z. Xiao Hong, "College teaching quality evaluation model based on BP neural network," Advanced Materials Research, vol. 591-593, pp. 2186-2189, 2012.

[11] X. Cheng, Z. Jin, and H. Yang, "Optimal insurance strategies: a hybrid deep learning Markov chain approximation approach," Astin Bulletin, vol. 50, no. 2, pp. 1-29, 2020.

[12] A. Sharififar, F. Sarmadian, and B. Minasny, "Mapping imbalanced soil classes using Markov chain random fields models treated with data resampling technique," Computers and Electronics in Agriculture, vol. 159, pp. 110-118, 2019.

[13] Y. C. Dong, L. Weidong, S. Hongtao et al., "Teaching eect assessment method of basis courses in engineering institutions based on homogeneous Markov chain," Journal of Mathematics Research, vol. 2, no. 1, p. 89, 2010. 
[14] J. Z. Zhu, J. L. Jianhui, and Z. H. Zhilei, "Multi-level fuzzy comprehensive evaluation on the classroom teaching quality of sports theory," International Journal of Digital Content Technology and Its Applications, vol. 7, no. 2, pp. 467-474, 2013.

[15] X. Cao, "Application of Markov chain analysis method to evaluate sports teaching quality," Applied Mechanics and Materials, vol. 543-547, pp. 4690-4693, 2014.

[16] X. Zheng, "Research on teaching efficiency of cost software based on the Markov chain," in Proceedings of the 2016 International Conference on Smart Grid and Electrical Automation (ICSGEA), pp. 71-75, Zhangjiajie, China, August 2015.

[17] X. F. Wang and X. Q. Wang, "The application research in the evaluation of sports teaching quality based on the analytic hierarchy process," Applied Mechanics and Materials, vol. 155-156, pp. 143-147, 2012.

[18] X. Zhang and Z. Jun, "Notice of retraction application of homogeneous Markov chain in quantitative analysis of teaching achievement indicators in physical education," in Proceedings of the 2010 Second International Workshop on Education Technology and Computer Science, pp. 530-532, Wuhan, China, March 2010.

[19] X. Lv, N. Li, X. Xu et al., "Understanding the emergence and development of online travel agencies: a dynamic evaluation and simulation approach," Internet Research, vol. 30, no. 6, pp. 1783-1810, 2020.

[20] N. Gao, D. Luo, B. Cheng, and H. Hou, "Teaching-learningbased optimization of a composite metastructure in the 0-10 $\mathrm{kHz}$ broadband sound absorption range," The Journal of the Acoustical Society of America, vol. 148, no. 2, pp. EL125EL129, 2020.

[21] B. Wang, F. C. Zou, J. Cheng et al., "Fault detection filter design for continuous-time nonlinear Markovian jump systems with mode-dependent delay and time-varying transition probabilities," Advances in Difference Equations, vol. 2017, no. 1, pp. 1-23, Article ID 262, 2017.

[22] J. Yang, J. Zhang, and H. Wang, "Urban traffic control in software defined internet of things via a multi-agent deep reinforcement learning approach," IEEE Transactions on Intelligent Transportation Systems, vol. 22, no. 6, pp. 37423754, 2021.

[23] C. Müller, H. Diedam, T. Mrziglod, and A. Schuppert, "A neural network assisted Metropolis adjusted Langevin algorithm," Monte Carlo Methods and Applications, vol. 26, no. 2, pp. 93-111, 2020.

[24] K. Sim, J. Yang, W. Lu, and X. Gao, "MaD-DLS: mean and deviation of deep and local similarity for image quality assessment," IEEE Transactions on Multimedia, 2020, In press.

[25] F. Zhang, W. Fang, C. Zhou, and Z. Liu, "A brief study on teaching evaluation system based on fuzzy rule with scientific teaching materials," in Advances in Computer Science, Environment, Ecoinformatics, and Education, pp. 99-105, Springer, Berlin, Germany, 2011. 